\title{
Polysèmes
}

Revue d'études intertextuelles et intermédiales

\section{Le «tournant exceptionnel » de Stanley Spencer : autoportraits et doubles portraits}

Stanley Spencer's Exceptional Turn: Self-portraits and Double Portraits

\section{Liliane Louvel}

\section{(2) OpenEdition}

1 Journals

Édition électronique

URL : http://journals.openedition.org/polysemes/8248

DOI : $10.4000 /$ polysemes. 8248

ISSN : 2496-4212

Éditeur

SAIT

\section{Référence électronique}

Liliane Louvel, « Le « tournant exceptionnel » de Stanley Spencer : autoportraits et doubles portraits », Polysèmes [En ligne], 24 | 2020, mis en ligne le 20 décembre 2020, consulté le 20 décembre 2020. URL : http://journals.openedition.org/polysemes/8248; DOI : https://doi.org/10.4000/polysemes 8248

Ce document a été généré automatiquement le 20 décembre 2020.

Polysèmes 


\title{
Le « tournant exceptionnel » de Stanley Spencer : autoportraits et doubles portraits
}

Stanley Spencer's Exceptional Turn: Self-portraits and Double Portraits

\author{
Liliane Louvel
}

\section{«An abnormal genius ?}

1 En août 1936, on pouvait lire dans un article du journal The Apollo «Stanley Spencer is a genius if an abnormal one » (cité dans Bell 162). En reconnaissant le génie de l'artiste, on en reconnaissait aussi le côté " anormal », dans le sens de ce qui fait exception à la norme, ce qui est l'exception. Le fait que le critique ait choisi comme critère définitoire appliqué à Spencer celui de l'anormalité est révélateur. D'une part, il assimile le caractère du peintre à sa production, confondant les deux. D'autre part, il lui reconnaît la qualité de génie qu'il module d'un bémol en usant du préfixe privatif. Or, rien n'est plus difficile à définir que la notion de normalité et, partant, d'anormalité, puisque lorsqu'il s'agit d'art, l'on se trouve dans le domaine du subjectif, et donc du goût. On s'avance aussi sur le territoire de l'invention, de l'innovation, voire, justement, de la transgression des normes. La notion de norme semble difficile à appliquer à une œuvre d'art à moins de se référer à un académisme poussiéreux et sclérosant. Les deux termes, " anormal » et "génie », sont pratiquement de nature oxymorique, car nul ne semble devoir être plus éloigné de la "normalité » que le "génie » (qui resterait à définir aussi). Les ouvrages ne manquent pas qui ont tenté de le faire. Mais la réaction du critique de l'Apollo semble refléter celle du public, plongé dans des abîmes de perplexité face aux œuvres singulières de Spencer dans le contexte des années trente.

C'est que les œuvres de Spencer exercent un effet étrange sur l'œil du spectateur. Se trouvant face à elles, l'œil se voit submergé, comme affolé parfois, par une multiplicité de détails, de figures et de scènes annexes, que seuls des peintres comme Brueghel l'Ancien et Jérôme Bosch (on pense au Jardin des délices) ou encore Uccello et 
Gozzoli (même si ses scènes sont là plus ordonnées et tranquilles) ou encore plus tard, les expressionnistes allemands, furent capables de créer. Ce travail semble témoigner d'un besoin obsessionnel de couvrir chaque centimètre carré de toile, souvent sous une fine couche de peinture (ceci pour des raisons plus financières qu'esthétiques), si bien que la texture fibreuse de la toile reste parfois apparente sous la couche de pigments. Face à ces toiles qui regorgent de créatures animées de mouvements agités et apparemment désordonnés, l'œil, comme déréglé, se perd et peine à faire son chemin et à trouver un parcours de vision.

Le besoin du peintre de multiplier les figures, les matières, les détails trouva souvent un espace de réalisation dans des triptyques complets avec prédelles, comme dans la peinture ancienne, voire dans des séries se développant sur des murs ou sur de longues pièces de toiles qu'il ne cessait d'augmenter. Cela semble témoigner d'un besoin d'engager l'ensemble de son corps, qui peut enfin trouver un espace à sa mesure dans l'emploi répétitif et le recours à la scansion temporelle des séries et des cycles qu'il réalisa dans les fresques des chapelles à Cookham, à Burghclere ou à Port Glasgow. On peut aussi discerner cette nécessité dans les séries du Christ dans le désert (The Disrobing of Christ, The Robing of Christ, Christ in the Wilderness) des années 1920-1930, ou encore celles des Béatitudes (fin des années 1930).

Lorsque l'on contemple les tableaux à figures des années 1933 à 1945, qui comportent des scènes du village de Cookham, des scènes domestiques et familiales avec Hilda, sa première épouse, et leurs deux fillettes, ainsi que la série des Béatitudes relatant la «passion » (il s'est bien agi de cela) de Spencer pour Patricia Preece, seconde épouse du peintre, ces «séries domestiques» («Domestic series») de Spencer dérangent et fascinent en même temps. On reste comme médusé devant ces scènes absolument originales, qui n'ont pas d'équivalent dans le monde de l'art, et qui témoignent cependant, ne peut-on s'empêcher de penser, de l'œuvre sinon d'un « ange du bizarre » du romantisme noir, comme E.A. Poe, du moins de celle d'un «artiste du bizarre». Certains critiques ne vont-ils pas jusqu'à classer Spencer dans la catégorie des néoromantiques?

5 Stanley Spencer ne manqua pas de défendre son point de vue en développant une crypto-théorie très personnelle de son art, que l'on peut reconstituer à partir d'éléments dispersés ici et là dans ses écrits. Il défendit son style, en particulier les distorsions pour lesquelles il était tant critiqué, et affirma fermement sa vision singulière de l'art et du monde, liant le spirituel et le temporel. «Pour moi le "tout" de l'Art est le Christ [...] le Christ prêcha l'Évangile de l'Art »'. Dans un essai intitulé «Distortion " paru en 1937-1938, il défendait, par exemple, le fait qu'un bras semblât trop long dans l'un des tableaux de la série des Béatitudes de l'Amour, intitulé Knowing et daté de 1938. Il s'agissait pour lui d'un problème de composition qui rendait nécessaire une telle déformation :

The arm is a part of the entire form of the picture and is perfectly placed and an integral part of the whole composition. It is not a question of altering the arm and making it the right length: it is a question of the degree of feeling in the first instance. The structure (namely the composition) holds everything it is meant to hold and the arm is the exactly right compositional shape in the structure of the composition. There is no altering it to the length we see it in nature by simply making it that natural length. If you do, away goes all the meaning of the picture: it would be as sensible as to destroy it altogether. (Spencer 187) 
Il s'agit là d'une vraie parole de peintre, s'exprimant en termes de problème pictural à résoudre et de solutions techniques à trouver pour produire les effets désirés, qui sont de l'ordre du visuel, du sensible et de l'intellect. Aucun compromis n'est envisageable et l'on doit se dégager de la vraisemblance et de la ressemblance aux « modèles » naturels, et donc s'éloigner de tout réalisme. On entend bien une parole originale, clairement mûrie et sûre de son bon droit, et qui fait vraiment exception. Dans un texte qu'il écrivit pour un recueil de sermons commandés à plusieurs artistes, dont Roger Fry luimême, par The Cockerel Press en 1934, Spencer indique :

An artist is not used to having to put a name to his feelings, but for himself this truth seems inseparable from such experiences as love, desire, faith, passion, intimacy, God, spiritual consciousness, curiosity, adventure, ingenuity. An artist wishes to absorb everything into himself [...] Distortion arises from the effort to see something in a way that will enable him to love it. It is unbearable for an artist to be continually seeing things in and through a film of apparent utter meaninglessness: he is engaged in a continual effort to remove the barrier. ${ }^{2}$

7 Si la Série Domestique et la série des Béatitudes, qui représentent des scènes conjugales ou amoureuses, sont frappantes, voire choquantes dans leur manière, elles font partie du domaine des œuvres privées. Parmi ces dernières, deux œuvres font exception deux fascinants doubles portraits de l'artiste et de sa femme qui sont tout à fait différents de ses autres œuvres. Non seulement elles divergent et innovent mais elles auront une descendance bien étonnante pour qui connaît l'ensemble de l'œuvre de Spencer. On pourra alors mesurer à quel point il fut un artiste inspiré dont le caractère exceptionnel allait être riche de potentialités futures, animées par une véritable Passion au sens amoureux du terme mais aussi christique dans une certaine mesure pour cet artiste qui liait ensemble amour, religion et art. Il s'agit d'œuvres peintes entre les années 1930 et 1940.

En guise d'introduction à la manière de peindre l'intime, il convient cependant de prêter d'abord attention aux autoportraits du peintre, seul - passage obligé de tout peintre qui se prend pour sujet premier toujours disponible-, puis aux doubles portraits et aux nus de lui-même avec sa seconde femme, avant d'aller jusqu'à l'exception de l'exception.

\section{Portraits autoportraits : quand l'artiste peint le peintre}

9 Tout au long de la carrière de Spencer, on voit passer la silhouette aisément reconnaissable du peintre qui apparaît de tableau en tableau. Elle fonctionne comme un indice de sa présence, constitue ce que Genette appelait un "effet de présence » (Genette 93) et peut être assimilée à une sorte de métalepse ou de leitmotiv. On sait à quel point Spencer aimait la musique - son père était l'organiste du village et fugues, variations et motifs récurrents sont souvent utilisés comme métaphores de son propre travail.

On voit alors revenir régulièrement un homme de petite taille, les cheveux bruns coupés au bol avec une frange et souvent vêtu d'un costume de tweed brun (ou d'un uniforme pendant la guerre), dépeint dans des scènes de sa vie quotidienne avec sa (ses) femme(s), ses enfants et les gens du village. C'est le cas des séries comme la Série domestique ou celle des Béatitudes. Il apparaît également dans ces scènes avec figures dont le thème est emprunté aux textes sacrés, comme dans des scènes de résurrection 
ou dans The Marriage at Cana: Bride and Bridegroom (1952) ou The Temptation of Saint Anthony (1945).

11 D'une certaine manière, Spencer donnait ainsi à voir une version fictionnalisée de sa vie intime et familiale, développant de manière visuelle ce qu'il disait souhaiter faire, c'est-à-dire écrire son autobiographie, ce qu'il ne parvint jamais à faire. C'est son petitfils, John Spencer, qui a repris le projet et publié le premier tome d'une biographie de Spencer, se servant des écrits autobiographiques épars laissés par le peintre, entre autres sources.

Les figures peintes que Spencer affectionnait particulièrement déploient un récit sous les yeux du spectateur. Elles s'enlacent, s'embrassent, s'étendent sur le sol, discutent, effectuent divers travaux dans les maisons, au village. Elles nourrissent, soignent, pansent les soldats, pendant la guerre. La petite silhouette du peintre est visible près des tentes, calmant les blessés étendus sur les brancards, prêts à être opérés dans l'hôpital de campagne à Smol en Macédoine. On le voit aussi dans la grande Résurrection de la Tate (1924) en compagnie de Richard Carline et de Hilda, étendu entre les deux dalles de pierre d'une tombe. S'il figure dans cette scène, c'est pour signifier qu'il partageait la joie de cette communauté unie dans la résurrection des corps. On ne s'étonnera pas de la puissance de ce désir puisque ces jeunes gens étaient de véritables «revenants » ayant survécu à la catastrophe de la Première Guerre mondiale. Le tableau exprime aussi le thème de la hantise de l'image et de son pouvoir de revenance, ces « images survivantes $»^{3}$ restant indélébiles dans la mémoire des soldats traumatisés. En se représentant dans ces scènes, Spencer avait peut-être cherché là un moyen d'exorciser l'horreur de la guerre.

\section{Sir Stanley Spencer and Mr Cookham}

13 N'hésitant pas à mêler sa vie et son œuvre créatrice, Spencer devait peindre un certain nombre d'autoportraits. Ce faisant, il organisait une sorte de documentation de sa propre vie, l'inscrivant ainsi dans le temps. C'est le cas de son portrait de 1914 (Tate Britain) (Fig. 1), dans lequel il regarde fixement son image dans le miroir et, à travers lui, le spectateur. On retrouve le même dispositif classique dans les portraits de 1924 où il regarde de trois-quarts, et ensuite dans les portraits de 1936, 1944, 1951 et 1959.

Dans l'autoportrait daté de 1959 (Tate Britain) (Fig. 2), Spencer montre le processus du vieillissement alors qu'il se trouve au seuil de la mort. Atteint du cancer, opéré puis de nouveau atteint, il peint chez lui ce portrait avant de retourner mourir au Cliveden Canadian Memorial Hospital (1959). Par une ironie du sort, cet hôpital militaire fut le dernier lieu des soins dispensés à celui qui avait prodigué les siens à l'hôpital militaire de Bristol, puis au front d'Orient.

Ce qui frappe dans ces portraits est la manière dont il fixe intensément le spectateur, comme pour l'engager dans un dialogue. Depuis l'espace représenté, il s'adresse à l'extérieur, au hors-cadre. Certains de ses portraits pourraient s'appeler «portraits de biais ", puisque le peintre semble se défier du miroir et du spectateur. C'est le cas des portraits de 1924, 1939 et 1951, dans lesquels on peut voir apparaitre des encadrements de tableaux derrière le peintre. Dans son Autoportrait de 1939 (Fitzwilliam Museum, Cambridge) (Fig. 3), il s'est représenté avec les instruments de son travail, mettant en avant sa palette et son pinceau. À l'arrière du portrait, Spencer a représenté les draps froissés du lit déserté par sa seconde femme, Patricia Preece. Ainsi, l'artiste semble 
vouloir montrer qu'il continue à peindre en dépit de tout, envisageant le tableau comme un défi. La main et la palette venant en avant du tableau semblent insister sur le savoir-faire du peintre, artiste et artisan de la couleur avant tout. Le pinceau pointe vers le haut, comme une flèche, une affirmation. L'étude des plis du lit n'est-elle pas aussi une manière de démontrer qu'il pouvait peindre à la manière des exercices académiques d'études documentaires et de "rendus » de drapés et de plis? Mais dans ce portrait, le travail des ombres sur le visage et sur la main accentue les effets de lumière, y compris dans la projection des lunettes fortement marquées sur le visage.

La manière dont sont peints ces portraits semble déjà annoncer la manière d'un peintre exceptionnel qui allait venir ensuite. Dans ses portraits et ses autoportraits, Lucian Freud utilise un traitement similaire des ombres et des lumières qui accentue les traits du visage grâce à une forte lumière zénithale. Dans l'un de ses portraits, intitulé Reflection (Self-Portrait) et daté de 1985 (collection particulière), Freud joue sur le double sens en anglais de reflet et de réflexion. Vient ensuite la confirmation de l'autoportrait.

Se représentant plusieurs fois (en 1939, 1951 et 1959), avec ses outils de travail ou devant le cadre d'un tableau, Spencer souhaitait aussi indiquer qu'il dotait son portrait d'une dimension réflexive et méta-réflexive. Ces portraits sont frappants dans leur manière d'interpeler le spectateur : ils semblent relever d'un dialogue muet à deux voix "mouchetées ", comme on le dit de fleurets subtils. Si le portrait de 1939 annonce ceux de Lucian Freud, avec son rendu bleuté des draps et la touche de la peinture, l'intuition sera confirmée par l'étude des doubles portraits du peintre avec sa seconde femme, peints à la même période. Car Lucian Freud peignit lui aussi de nombreux doubles portraits, comme celui d'une femme et d'un chien étendus et endormis (Double Portrait de 1985-1986) ou celui d'un homme et d'une femme intitulé And the Bridegroom de 1993, entre autres.

Dans certains des tableaux effectués autour des années trente à une période pendant une période cruciale et déchirante de sa vie, Spencer alla au-delà de la simple représentation de lui-même. Il produisit des Nus doubles (Double Nude Portraits) absolument novateurs, vraiment exceptionnels dans tous les sens du terme.

\section{Portraits, autoportraits et doubles portraits}

19 Autour des années 1930, Spencer fit la connaissance de Patricia Preece, artiste peintre qui gravitait dans le cercle de Bloomsbury. Elle vint s'installer à Cookham avec sa compagne Dorothy Hepworth, peintre elle-même. Spencer, fasciné, s'éprit d'elle et souhaita avoir une relation amoureuse avec elle tout en conservant Hilda et sa famille. Hilda refusa et s'éloigna de lui. Il épousa Patricia Preece qui, pour leur lune de miel, partit en Cornouailles avec son amie, invitant Spencer et même Hilda à les rejoindre. Cette union fut une catastrophe à tous points de vue. Patricia Preece saisit l'occasion d'exploiter l'engouement de Spencer pour exiger de nombreux cadeaux onéreux, des bijoux ou des vêtements qui le ruinèrent et le forcèrent à produire de plus en plus de paysages qui se vendaient bien mais qu'il disait détester réaliser. Il fit don à Patricia de la maison achetée avec Hilda et dut quitter les lieux lorsqu'ils divorcèrent. Hilda sombra dans une grave dépression et fut internée dans un hôpital où Spencer lui rendit visite jusqu'à sa mort en 1950. Il continua même à lui écrire au-delà de sa mort, ce dont témoignent plusieurs œuvres et écrits. "In the morning, on the sun blazing hot road, we had the midnight of our desire ", écrivait-il à Hilda en 1948 (cité dans Spencer 130). 
L'expression rappelle "the winter of our discontent", "l'hiver de notre mécontentement», citation extraite des deux premiers vers de Richard III de Shakespeare : "Now is the winter of our discontent / Made glorious summer by this sun [or son] of York». Ces mots évoquent clairement la séparation difficile du couple brisé par la passion de Spencer.

Les épreuves et les problèmes conjugaux que subit Spencer dans les années 1935-1937 semblent avoir infusé ses tableaux, transformant profondément sa manière de peindre. De son union catastrophique avec Patricia Preece et de cette époque tourmentée subsistent les œuvres qui ont parfois été appelées " the sex pictures », tableaux de nus de Patricia Preece et doubles portraits de l'artiste et de sa femme, nus également. Ils sont d'une facture tout à fait différente du reste de l'œuvre. Ils reflètent sa détresse, sa frustration et son incompréhension face au pouvoir du sexe et au désir qu'il avait peine à intégrer à sa vision intime. Il conserva certains de ces tableaux sous son lit jusqu'à sa mort, ayant conscience de leur exceptionnel pouvoir subversif.

Les portraits de Preece seule, vêtue ou semi-vêtue pour l'un d'entre eux, arrêtent le spectateur, qui est comme appelé par le regard intense du modèle fixé sur le peintre, dont on devine la présence hors cadre, et par conséquent sur le spectateur. C'est le cas de Nude Portrait of Patricia Preece (1935), et de Nude (Portrait of Patricia Preece) or Girl Resting (1936). La texture de la peinture déploie un blanc, un bleu, un brun et un rose qui semblent iridescents. La matière est utilisée à larges touches et dans une manière plus réaliste qui contraste avec la façon dont Spencer rendait les figures dans ses œuvres d'imagination ou dites «illustratives». Nulle concession à la beauté traditionnelle ni à l'idéalisation : les seins du modèle sont lourds et pendent sur le côté dans le tableau de 1935. Distendus, ils frôlent sa taille. Des poils remontant du pubis dessinent comme un triangle qui monte jusqu'au nombril. Un autre triangle inversé, sur le décolleté, indique les traces d'une exposition au soleil. Dans le second tableau, le modèle est vêtu d'un sous-vêtement noir dont la dentelle laisse apparaître l'auréole des seins. Le pied droit du modèle est coupé par le cadre du tableau et les doigts de sa main gauche sont pris entre ses doigts de pied. Un travail précis sur le rendu des draps, de leurs plis et de leurs froissements, relève de l'étude documentaire parfaitement maîtrisée. On retrouve cela dans les deux doubles portraits Self-Portrait with Patricia Preece de 1936 et Double Portrait with Patricia Preece (1937), qui confirment l'évolution de son style à cette époque. Le sujet classique du peintre et de son modèle a donné naissance ici à ces deux tableaux singuliers, exceptionnels par leur facture et par leur originalité au sein de l'œuvre de Spencer.

Car ce qui frappe d'abord dans ces œuvres comme dans les portraits et nus de Patricia Preece, c'est le changement radical de la facture des tableaux et de la manière de peindre de Spencer puisque les deux doubles portraits sont des études de nus plus "réalistes». Les détails anatomiques relevés plus haut le prouvent. Les corps sont présentés en très gros plans et exprimés dans des tons de mauve, de bleu et d'un vert glauque. La chair opalescente est traitée à larges coups de brosse sans efforts pour dissimuler la matière picturale. Celle-ci semble même rivaliser avec le sujet, soulignant ainsi la facture et le faire $d u$ peintre. Ces deux œuvres mettent en scène une interrogation métaphysique et l'on voit comment la peinture peut penser par ses moyens, en choisissant comme sujet la pensée et la vision d'une double interrogation.

Dans la première œuvre, datée de 1936-1937 (Fig. 4), l'artiste apparaît en gros plan mais vu de dos, comme coupé à hauteur des omoplates et tout proche du plan 
représentant. Il semble être debout face à la scène, au lit, et il se tient entre le spectateur et le sujet du tableau. Lunettes sur le nez, il regarde de profil son modèle, servant ainsi de relais et de délégation des regards : le spectateur regarde le tableau, regarde le peintre de dos, qui regarde le modèle. La femme est allongée sur le côté, et elle regarde ailleurs, avec une sorte de regard un peu rêveur cette fois. Tous deux sont peints en très gros plan et occupent tout l'espace de la toile. Aucune concession n'est faite à la beauté idéale. Spencer porte ses lunettes, comme pour mieux voir et peindre cette scène spectaculaire d'interrogation et d'étonnement.

Dans un autre double portrait de 1937, que l'on appelle aussi The Leg of Mutton Nude

(Fig. 5), Patricia Preece est allongée au premier plan cette fois, les jambes écartées. Spencer est accroupi derrière elle, lui aussi jambes écartées. Il porte ses lunettes et contemple le corps de celle qui était alors sa femme sans l'être vraiment. Derrière eux, un poêle, peint avec minutie, rougit ses vitres. L'arrière-plan n'est jamais banal chez Spencer. On a vu que dans le premier double portrait, c'est le lit défait avec les draps soigneusement peints et le papier peint à fleurs qui, comme chez Vuillard, forment une sorte de tapisserie d'accompagnement. Spencer portait une attention aux détails touchant à l'obsession, et l'on retrouve un autre poêle qu'il insista de peindre à l'arrière-plan du Portrait of J. E. Martineau (1956), qui est richement vêtu d'un lourd manteau à col de fourrure à la Holbein et arbore l'insigne or et émail de ses fonctions honorifiques. Dans le fonds des Musées nationaux d'Écosse (National Galleries of Scotland, Édimbourg) on peut également voir le magnifique portrait... d'un feu, Fire Alight de 1936, avec une grille en forme de harpe où l'on peut aussi lire deux S en vis-àvis qui rappellent les initiales du peintre et entourent des barreaux dont l'un est brisé.

Au tout premier plan de Double Portrait with Patricia Preece, ou The Leg of Mutton Nude, se trouve la chair écorchée d'un gigot de mouton prêt pour la cuisson et une côtelette, comme allongés contre elle et orientés dans le même sens que ses cuisses ${ }^{4}$. La main de Patricia Preece proche du gigot insiste sur la proximité entre les chairs et la viande : on a l'impression que ses deux cuisses et celle de l'animal sont mises en parallèle. Ce tableau de chairs aux tons bleutés, roses, bruns et rouges (dans un camaïeu qui décline la brique du mur derrière, le feu et la viande de mouton) rappelle la vanité de toute chose, cette "vanité des vanités " de l'Ecclésiaste. Il vient aussi renouveler les grands tableaux de boucherie et les bœufs écorchés de Rembrandt ou de Soutine. Ce tableau suscite une interrogation profonde sur le corps, sur l'attente et les surprises du plaisir, de la frustration et de la souffrance, ou encore sur les désenchantements de la sexualité, les pièges du désir et la trivialité de la condition humaine. C'est une évocation de la tristesse de l'amour « charnel ", pour utiliser le langage religieux, de la tromperie des apparences et des abîmes de perplexité dans lesquels ces tourments peuvent plonger l'être humain. En outre, ce tableau d'un homme qui aurait voulu garder ses deux femmes introduit dans la toile même son désir de l'une et la présence de l'absente. "The Leg of Mutton" désignait un pub près d'un étang où le couple qu'Hilda et Spencer formaient dans leur jeunesse avait l'habitude de se rendre à l'époque du « minuit de leur désir » (« the midnight of their desire »). Ces éléments font de ce tableau un rébus visuel ou une énigme et en confirment la valeur métaphysique. Spencer redonnait ainsi métonymiquement à Hilda une seconde présence dans le tableau de son nouveau couple. Le pub et son nom évoquent des souvenirs du passé, sous l'égide de l'un des lieux emblématiques préférés des Britanniques faisant partie de leur identité nationale. L'autoportrait du peintre nu qui ne dissimule rien de son anatomie n'est pas sans rappeler l'autoportrait de Dürer exécuté à la plume et au 
pinceau et conservé à Weimar (1500-1512). Dürer, qui a peut-être été l'un des premiers à peindre plusieurs autoportraits, s'y est représenté entièrement nu, debout face au spectateur, comme l'interpellant du regard. L'œuvre est une étude de muscles et de chairs, un travail sur la connaissance du corps humain et sur sa fragilité. Dans le travail de Spencer sur la variété des couleurs des chairs opalescentes ou sur la disposition des corps étendus peints sans concession au bon goût, qui témoignent plutôt d'un goût du morbide assorti d'une "inquiétante étrangeté " parfois repoussante, on peut encore retrouver une parenté avec les portraits et les nus qui seront peints un peu plus tard par Lucian Freud, en particulier, l'autoportrait intitulé Reflection de 1985 (voir plus haut), tout en nerfs et en tendons, ou le nu Standing by the Rags de 1988-1989.

Les deux autoportraits semblent mettre en scène l'interrogation fondamentale de l'homme confronté à la totale altérité de la femme et à l'influence perturbatrice de la sexualité lorsqu'elle fait irruption dans la vie quotidienne et la bouleverse totalement. Ce sont là deux œuvres extraordinairement bien peintes, comme Spencer lui-même le reconnaissait, et profondes. On peut avancer que Spencer parvint à représenter la pensée en train d'avoir lieu tout en peignant la chair. Il alliait la quête de la spiritualité qu'il tenta toute sa vie d'atteindre et ses difficultés à y parvenir, comme empêché dans la prison inévitable de son propre corps et de ses désirs. Sa découverte de la puissance de la sexualité, capable de transformer un être à son corps défendant, ne fut pas la moindre des expériences difficiles auxquelles fut alors soumis Spencer. La chair traitée comme la viande montre aussi verticalement, en quelque sorte, ce qu'il en est d'être humain. D'autres artistes, discrètement mais non moins efficacement, suivront le même chemin: Lucian Freud, mais aussi son ami Francis Bacon, dont les tableaux montrent l'écoulement des chairs hors des diagrammes qui tentent de les contenir et de l'armature de ces triptyques dont il dresse les structures anciennes et apotropaïques.

La vision de Spencer est donc celle d'un artiste singulier qui n'hésitait pas à mettre en scène sa propre vie et celle des habitants de son village, introduisant au sein de lieux familiers et dans son propre foyer le trouble d'une inquiétante étrangeté. Se dégage également de ces tableaux des années 1930 une atmosphère de conte cauchemardesque (Spencer lut Andersen toute sa vie), quelque chose de semblable à ce qu'Angela Carter en littérature (qui révise les contes dans The Bloody Chamber par exemple) et Paula Rego en peinture firent en tordant et remodelant la cruauté fantastique. Les contes inquiétants façonnés à partir de scènes domestiques effectuent une métamorphose de l'ordinaire en une exceptionnelle expérience visuelle.

\section{BIBLIOGRAPHIE}

Bell, Keith. Stanley Spencer: A Complete Catalogue of the Paintings. London: Phaidon Press, 1992.

Deleuze, Gilles. Francis Bacon, logique de la sensation. Paris : Éditions du Seuil, « L'ordre philosophique ", 2002. 
Didi-Huberman, Georges. L'Image survivante : histoire de l'art et temps des fantômes selon Aby Warburg. Paris : Éditions de Minuit, « Paradoxe », 2002.

Genette, Gérard. La Métalepse. Paris : Éditions du Seuil, 2004.

Spencer, John (ed.). Stanley Spencer Looking to Heaven. London: Unicorn Press, 2016.

Spencer, Stanley. Stanley Spencer: Letters and Writings. Adrian Glew (ed.) London: Tate Publishing, 2001.

\section{Liste des figures}

Fig. 1 : Autoportrait (1914), huile sur toile, 63 x $51 \mathrm{~cm}$, Tate Britain.

Fig. 2 : Autoportrait (1959), huile sur toile, 50,8 × 40,6 cm, Tate Britain.

Fig. 3 : Autoportrait (1939), huile sur toile, 39,7 x 55,2 cm, The Fitzwilliam Museum, Cambridge.

Fig. 4 : Self-Portrait with Patricia Preece (1937), huile sur toile, 61 x 91,2 cm, The Fitzwilliam Museum, Cambridge.

Fig. 5 : Double Portrait with Patricia Preece (1937), huile sur toile, 83,8 x 93,7 cm, Tate Britain.

\section{NOTES}

1. "To me the 'whole' of Art is Christ [...] Christ preached the Gospel of Art; his words and acts are an exposition of the three great laws of Art: charity, faith and love », cité dans Glew 98.

2. «Sermons by Artists», Stanley Spencer, The Cockerell Press, 1934, Notebook 1934 (manuscript version) 8055-44, cité dans Glew 165.

3. Georges Didi-Huberman, L'Image survivante: Histoire de l'art et temps des fantômes selon Aby Warburg, Paris, Les Éditions de Minuit, coll. Paradoxe, 2002.

4. Spencer semble s'y être référé en l'appelant « le gigot non cuit », peut-être en référence à la non-consommation de son union avec Patricia Preece.

\section{RÉSUMÉS}

Stanley Spencer est bien connu pour ses scènes souvent inspirées des Saintes Écritures, souvent situées dans son village natal de Cookham dans le Berkshire. Très critiqué à son époque pour ces scènes, jugées trop illustratives par Roger Fry et dont les personnages semblent désarticulés, avec des membres distordus, et une agitation frénétique, il n'en marqua pas moins son époque. Parmi les nombreuses œuvres de sa production picturale, les années trente marquent un changement important dans le choix des sujets. La pâte devient plus épaisse, les couleurs plus claires et nuancées, les personnages sont peints en gros plan. Il s'agit d'autoportraits et surtout de doubles portraits de nus avec celle qui était sa seconde femme à l'époque, Patricia Preece. Ces œuvres datent d'une époque particulièrement douloureuse pour le peintre. Le changement de manière est frappant également dans la mesure où il annonce certains des peintres de la génération suivante, tel Lucian Freud, qui allait lui aussi peindre des nus avec une pâte épaisse 
dans des postures jugées particulièrement indécentes pour les critiques et le public. Les «sex pictures » de Spencer telles qu'elles furent nommées ouvraient ainsi la voie à l'École de Londres tout en témoignant de la manière dont l'art subit les tourments de la vie.

Stanley Spencer is well-known for his scenes inspired by the Scriptures, often located in Cookham, Berkshire, his native village. Receiving a very critical reception in his time because his scenes were deemed too illustrative by Roger Fry, for instance, and his characters shocking with their disjointed members when launched in a frenzied agitation, he was nevertheless recognized for his talent in his own time. Among the numerous pictures he painted, those achieved in the thirties display a radical turn in the astonishing manner and choice of subjects he adopted. The matter is thicker, the colours clearer and with nuances, the characters are painted with heavy layers and the subjects stand or lie at close range in the foreground. They are nude self-portraits and double nude portraits of himself and his then second wife, Patricia Preece. These paintings correspond to a particularly agonizing period for the painter. The change in his manner also heralds some of the painters of the next generation. For instance, Lucian Freud was going to apply thick layers of paint to his models often lying in particularly indecent poses as judged by both critics and public. Spencer's "sex pictures", as they were called, were thus paving the way for the London School while testifying to the way art may be informed by one's life's torments.

\section{INDEX}

Mots-clés : portrait, autoportrait, nu, Cookham, distorsion, tournant radical

Keywords : portrait, self-portrait, nude painting, Cookham, distortion, radical turn oeuvrecitee Domestic Series, the Beatitudes of love

\section{AUTEURS}

\section{LILIANE LOUVEL}

Liliane Louvel est professeur émérite à l'Université de Poitiers. Son champ de recherche est la littérature britannique contemporaine et les rapports texte/image. Elle a publié cinq ouvrages sur cette problématique : L'Eil du texte (PU du Mirail, 1998), The Picture of Dorian Gray, Le double miroir de l'art (Ellipses, 2000), Texte/image, images à lire et textes à voir (PUR, 2002), Le Tiers pictural, Pour une critique intermédiale (PUR, 2010). Elle a également dirigé plusieurs recueils d'essais ou ouvrages collectifs sur ce sujet : «Word/image », numéro spécial de EJES ; Like Painting (publication de La Licorne, Poitiers), Texte/image : nouveaux problèmes avec Henri Scepi (PUR, 2005), Intermedial Arts, Disrupting, Remembering and Transforming Media avec Leena Eilitta et Sabine Kim (Cambridge Scholars Press, 2012), Musing in the Museum, avec Laurence Petit et Karen Brown (Word/Image, Taylor and Francis, 2015). Elle prépare actuellement un ouvrage sur Stanley Spencer pour Sorbonne Université Presses. 